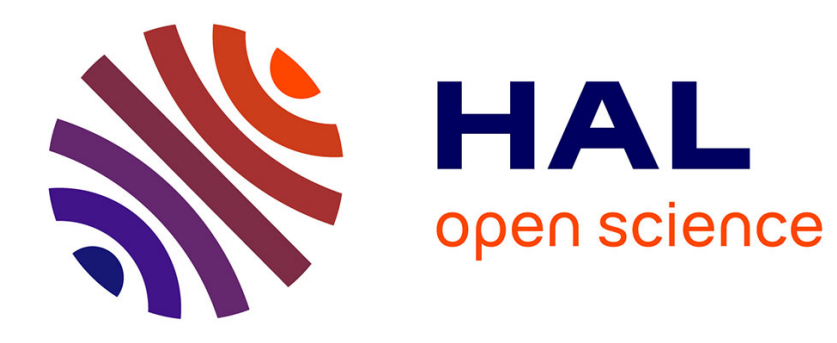

\title{
Réduire la Révolution en système ?
}

Olivier Ritz

\section{To cite this version:}

Olivier Ritz. Réduire la Révolution en système ?. Sophie Marchand, Élise Pavy-Guilbert. L'Esprit de système au XVIIIe siècle, Hermann, pp.131-141, 2017, 10.3917/herm.march.2017.01.0131 . halshs03372864

\section{HAL Id: halshs-03372864 \\ https://shs.hal.science/halshs-03372864}

Submitted on 11 Oct 2021

HAL is a multi-disciplinary open access archive for the deposit and dissemination of scientific research documents, whether they are published or not. The documents may come from teaching and research institutions in France or abroad, or from public or private research centers.
L'archive ouverte pluridisciplinaire HAL, est destinée au dépôt et à la diffusion de documents scientifiques de niveau recherche, publiés ou non, émanant des établissements d'enseignement et de recherche français ou étrangers, des laboratoires publics ou privés. 


\section{RÉDUIRE LA RÉVOLUTION EN SYSTÈME ?}

La Révolution française a suscité d'innombrables comptes rendus et commentaires. Les textes témoignent, accusent ou défendent : ils participent pleinement au débat politique du temps. Relais ou contre-points des discours d'assemblée, les ouvrages imprimés démultiplient le débat sur la Révolution qui commence dès 1789 et qui n’est mis en sourdine que lorsque Napoléon assoit définitivement son pouvoir, dans les premières années du XIX ${ }^{e}$ siècle. Si ces textes expriment les désordres révolutionnaires, multipliant par exemple les métaphores naturelles comme "effervescence », "commotion » ou « orages », ils constituent aussi des tentatives de remettre de l'ordre. Le récit des faits restitue un ordre chronologique en présentant un déroulement linéaire du processus révolutionnaire. Les dictionnaires qui établissent la liste des victimes de la Révolution ${ }^{1}$ ou celle des hommes qui ont joué un rôle politique construisent un ordre biographique ${ }^{2}$. Les textes qui aspirent à un ordre scientifique vont plus loin. Envisageant la nature comme un désordre apparent que l'on peut ramener à l'ordre si l'on en découvre les lois, ils font des sciences de la nature le modèle qui permettrait de réduire la Révolution en système, c'est-à-dire de l'expliquer par des éléments simples et liés les uns aux autres. Le tableau logique est l'expression la plus achevée de cette ambition systématique: souvent annoncé, parfois réalisé, il traduit la volonté de faire apparaître toute la logique de la Révolution en un coup d'œil ${ }^{3}$.

Est-ce à dire que le désordre révolutionnaire a entraîné, sur le terrain d'une science politique qui s'invente et se cherche, un retour en grâce de l'esprit de système ? Le projet de réduire la Révolution en système est très largement exprimé par des auteurs que tout semble opposer. Les raisons de cette vogue sont contradictoires : au prestige des sciences naturelles qui influence tous les auteurs s'ajoutent l'engagement rationaliste des uns et plus encore les partis pris conservateurs des autres. Pourtant, ces projets sont rarement menés à bien : le désir de système est contrebalancé par un renouveau de la critique de l'esprit de système.

Parmi les nombreux textes qui prétendent rendre compte des événements révolutionnaires à partir de 1789 , certains attirent l'attention par leur systématisme poussé à l'extrême. L'un de ceux qui va le plus loin est La Grande Période ou Le Retour de l'âge d'or de Jean Delormel. La Révolution est intégrée à une histoire de l'humanité entièrement expliquée par un principe unique : la « révolution totale et orbiculaire de l'écliptique ${ }^{4}$ ». Autrement dit, l'inclinaison de l'axe de rotation de la terre varie de façon régulière, décrivant elle aussi un cercle. Les climats et les saisons évoluent en conséquence. Le déluge correspond au moment où, l'axe de rotation de la terre étant aligné avec l'orbite du soleil, les glaces du pôle exposé ont fondu. Au contraire, lorsque cet axe est parfaitement

\footnotetext{
${ }^{1}$ Louis-Marie Prudhomme, Histoire générale des crimes commis pendant la Révolution française, Paris, an V-1797. Le tome second a pour titre «Dictionnaire des individus envoyés à la mort judiciairement, révolutionnairement et contrerévolutionnairement, pendant la révolution, particulièrement sous le règne de la convention nationale ».

2 Jean-Luc Chappey, Ordres et désordres biographiques. Dictionnaires, listes de noms, réputation des Lumières à Wikipédia, Seyssel, Champ Vallon, 2013.

3 Ce sens du mot tableau, très éloigné des sens pictural et théâtral, pouvant s'appliquer au «Système figuré des connaissances" de l'Encyclopédie, est très présent dans les textes de la période révolutionnaire. Pourtant, il ne sera enregistré que dans la sixième édition du Dictionnaire de l'Académie en 1832-1835 : «Il se dit également d'Une feuille, d'une planche sur laquelle des matières didactiques, historiques et autres, sont rédigées et rangées méthodiquement, pour être vues d'un coup d'œil ».

${ }^{4}$ Jean Delormel, La Grande période ou Le Retour de l'âge d'or, Paris, Blanchon et Belin, 1790, p. 23.
} 
perpendiculaire à l'orbite du soleil, les variations saisonnières n'existent plus et les régions tempérées jouissent d'un printemps perpétuel. Au moment où Delormel écrit, l'axe de rotation se redresse très progressivement, conduisant l'humanité vers un nouvel âge d'or dont la Révolution est l'un des signes annonciateurs : «La révolution qui s'opère depuis en Europe, et spécialement en France, devient une preuve de [notre] théorie ${ }^{5}$. Grâce à des figures gravées, insérées entre l'introduction et le premier chapitre, le lecteur «entendra aisément tout le système ${ }^{6} \gg$.

La théorie existait déjà partiellement chez le chevalier de Louville et chez l'abbé Pluche ${ }^{7}$. L'utilisation du contexte révolutionnaire pour la faire valoir est nouvelle. Delormel publie trois fois son texte : en 1790 puis en 1797 et 1805. La seconde édition, dont les planches sont identiques à la première, a pour titre Les Causes et les Époques des révolutions du monde physique et moral, on la grande période solaire ${ }^{8}$. Delormel essaie ainsi de tirer profit du succès éditorial promis à tous les textes qui expliquent la ou les révolutions pour faire valoir sa théorie. En faisant de la Révolution une preuve qui s'ajoute aux preuves des sciences et de la littérature ancienne, toutes convergentes ${ }^{9}$, il l'intègre à un système d'explication du monde où tout dépend d'un principe astronomique unique.

Le Coup d'ail sur le mécanisme et l'objet des révolutions depuis 2600 ans, publié sans nom d'auteur en 1799, donne l'exemple d'un raisonnement similaire mais plus abstrait et plus systématique dans sa présentation. Partant de la régularité du monde naturel, prouvée par les cycles des astres, des saisons et des marées, le texte conclut dès les premières lignes à la régularité des «mouvements politiques » et à «l'unité de principes ». Dès lors, une «base universelle », c'est-à-dire une loi unique, permet d'expliquer toute l'histoire de l'humanité. La nature détruit l'être faible pour le remplacer par un être plus fort. «En appliquant les principes de vie et de mort aux événements politiques les plus compliqués, on aura leur explication en les réduisant ainsi à leur plus simple valeur ${ }^{10}$.» Une fois la démonstration achevée, l'ensemble du système apparait sous la forme d'un «Tableau de huit révolutions, divisées selon leurs degrés ", depuis la révolution «de Rome s'érigeant en République » jusqu'à celle « des Français s'érigeant en République ${ }^{11}$ ».

Ces deux textes, qui peuvent sembler une survivance de l'esprit de système, sont moins marginaux qu'il n'y parait au premier abord. Ils ne font que mener à bien un projet exprimé par bien des auteurs de premier plan. Dans son Esquisse d'un tableau bistorique des progrès de l'esprit humain, Condorcet entend «tracer avec quelque vraisemblance, le tableau des destinées futures de l'espèce humaine, d'après les résultats de son histoire ${ }^{12}$ ». Constant propose d'approfondir la science politique grâce à ce qu'il appelle le «système des principes »: "Tout dans l'univers a donc ses principes, c'est-à-dire, toutes les combinaisons, soit d'existences, soit d'événements, mènent à un résultat : et ce résultat est toujours pareil, toutes les fois que les combinaisons sont les mêmes. C'est ce résultat qu'on nomme principe ${ }^{13}$.» Il indique en conclusion de son texte que le système des principes

\footnotetext{
${ }^{5}$ Ibid., note 2 de la p. 2 , en fin de volume, p. 323.

${ }^{6}$ Planche insérée entre les p. 10 et 11.

7 Voir Jean Starobinski, «L'inclinaison de l'axe du globe », dans Jean-Jacques Rousseau, Essai sur l'origine des langues, Paris, Gallimard, «Folio essais », 1990, p. 165-189.

${ }^{8}$ Jean Delormel, Les Causes et les époques des révolutions du monde physique et moral, on La Grande période solaire, seconde édition, L'Auteur, Crapart et alii, an V-1797.

${ }^{9}$ Dans l'avis de la seconde édition il se félicite de «l'éloquente harmonie avec laquelle toutes les sciences lui paraissent concourir à la démonstration de cette précieuse découverte » (ibid.).

${ }^{10}$ Coup d'cil sur le mécanisme et l'objet des révolutions depuis 2600 ans, Paris, 1799, p. 3-4.

${ }_{11}$ Ibid., p. 126-127.

${ }^{12}$ Condorcet, Esquisse d'un tableau historique des progrès de l'esprit humain, Paris, Agasse, an III, p. 327-328.

13 Benjamin Constant, Des réactions politiques, [1797], dans De la force du gouvernement actuel de la France et de la nécessité de s'y rallier, Des réactions politiques, Des effets de la terreur, éd. de Philippe Raynaud, Paris, Flammarion, 1988, p. 132.
} 
permet « des calculs politiques, rapprochés des sciences exactes par leur précision ${ }^{14} »$. Dans De la littérature, Germaine de Staël demande de la même manière: "Pourquoi ne parviendrait-on pas un jour à dresser des tables qui contiendraient la solution de toutes les questions politiques, d'après les connaissances de statistique, d'après les faits positifs que l'on recueillerait sur chaque pays ${ }^{15}$ ? »

Ces affirmations sont celles des défenseurs du progrès, de ceux qui revendiquent et prolongent l'héritage du siècle des Lumières. La volonté d'établir des systèmes explicatifs et prospectifs est encouragée par les évolutions scientifiques et administratives de la période : Lavoisier révolutionne la chimie en la rendant plus systématique, la France est organisée systématiquement en départements et un système uniforme de poids et de mesures se met en place $^{16}$. La puissance des nouveaux systèmes est telle qu'elle peut encourager le volontarisme de la raison chez les auteurs qui entreprennent d'écrire la Révolution.

Pourtant, le projet d'une pensée systématique de la Révolution est encore plus présent chez les auteurs de la Contre-Révolution. Chateaubriand assure dans l'Essai sur les Révolution qu'il serait "possible de dresser une table, dans laquelle tous les événements imaginables de l'histoire d'un peuple donné, se trouveraient réduits avec une exactitude mathématique ». Dans une note assez folle, il imagine la forme que pourrait prendre un tel tableau :

L'homme naturel, l'homme politique et l'homme civil se trouveraient rangés sous deux colonnes; sur une troisième seraient marqués les degrés de lumières et d'ignorance ; sur une quatrième, les chances et les hasards. On multiplierait alors tous ces nombres par les différentes passions, comme l'envie, l'ambition, la haine, l'amour, etc., qu'on verrait écrites sur une cinquième colonne: tout cela tomberait en fractions composées, par des nuances des caractères, etc. ${ }^{17}$.

Ce développement d'inspiration mathématique ne fait pas du premier Chateaubriand un défenseur du progrès par les sciences. Au contraire, sa visée est conservatrice puisqu'il vise à démontrer l'impossibilité du progrès par la répétition du même : "L'homme, faible dans ses moyens et dans son génie, ne fait que se répéter sans cesse; [...] il circule dans un cercle, dont il tâche en vain de sortir ${ }^{18}$.»

Il faut interpréter de la même manière les tableaux comme celui que propose le Coup d'ail sur le mécanisme et l'objet des révolutions. Réduire les révolutions en système permet de refuser toute spécificité à la Révolution française. C'est sans doute la raison qui a poussé l'éditeur Michel à fabriquer un ouvrage composite où Chateaubriand se trouve malgré lui lié au Coup d'cil. L'ouvrage reprend la plus grande partie du titre de l'essai de Chateaubriand, publié à Londres en 1797 et quasi introuvable en France ${ }^{19}$. Il rassemble une traduction française de l'introduction des Annals of the French Revolution d'A.-F. Bertrand de Molleville et le Coup d'cil sur les mécanismes et l'objet des révolutions. Tout semble opposer ces deux textes: le premier est un abrégé de l'histoire de la Révolution ouvertement royaliste qui affirme que la Révolution française ne ressemble à aucune autre. Il est à l'opposé du systématisme du

\footnotetext{
14 Ibid., p. 152.

${ }_{15}$ Germaine de Staël, De la littérature considérée dans ses rapports avec les institutions sociales, [1800], éd. de Gérard Gengembre et Jean Goldzink, Paris, GF Flammarion, 1991, p. 368.

16 Voir Jean-Baptiste Biot, Essai sur l'histoire générale des sciences pendant la Révolution française, Paris, Duprat et Fuchs, an XI1803, p. 35 : «Parmi les grandes idées que réalisa cette première époque de la révolution, il faut compter celle d'un système uniforme de mesures ».

${ }_{17}$ Chateaubriand, Essai bistorique, politique et moral sur les révolutions anciennes et modernes, considérées dans leurs rapports avec la révolution française, [1797], éd. d'Aurelio Principato, Euvres complètes, vol. I-II, Paris, Champion, 2009, p. 1170.

18 Ibid.

${ }_{19}$ Essai bistorique, politique et moral sur les révolutions anciennes et modernes, jusques et y compris l'époque du 18 Brumaire an 8, précédé d'un abrégé raisonné de la révolution française, Paris, Michel, s.d [1801]. Sur ce texte, voir Pierre Riberette, "Enquête sur un faux Essai sur les révolutions », Bulletin de la Société Chateaubriand, nº 40,1997 , p. 28-31.
} 
second texte. Il s'agit en réalité de deux manières d'arriver au même but: défendre des partis pris conservateurs.

Les projets de systèmes politiques sont par conséquent tout aussi présents chez les penseurs de la Contre-Révolution que chez les défenseurs du progrès. Pour Joseph de Maistre, bien que le «mouvement politique» de la Révolution dépende avant tout de la volonté divine, il a ses lois qui peuvent être tirées de l'observation, sur le modèle des lois de la physique et de l'astronomie: "Si l'on avait des tables de massacres comme on a des tables météorologiques, qui sait si l'on n'en découvrirait point la loi au bout de quelques siècles d'observations ${ }^{20}$ ? 》 Nombre d'écrivains contre-révolutionnaires se voudraient ainsi des Newton des mœurs. La physique newtonienne - sans ses détails et ses apories - leur procure le modèle d'une régularité parfaite qu'ils peuvent appliquer aux hommes pour affirmer que toute velléité de nouveauté est vaine. C'est pourquoi les adversaires les plus acharnés du nouvel ordre des choses défendent volontiers une vision systématique de l'histoire humaine.

Malgré tant d'affirmations convergentes, les projets d'explication systématique de la Révolution ont rarement abouti : La Grande période et le Coup d'cil sur les mécanismes des révolutions apparaissent comme des exceptions. La plupart des auteurs ont rapidement pris de la distance avec les systèmes.

Les voix de ceux qui condamnent l'esprit de système en politique se font de plus en plus fortes. Dans les premières années de la Révolution, l'argumentation d'Edmund Burke repose déjà sur cette condamnation : les hommes qui gouvernent doivent être "purgés du levain des systèmes » alors que les ministres de Louis XVI ont été eux-mêmes «infectés de la contagion des projets et des systèmes ${ }^{21} »$. Le système est une maladie parce qu'il empêche de voir la réalité en lui substituant des abstractions. Burke condamne de la même manière les projets de constitution discutés à l'Assemblée nationale: les révolutionnaires français ont de la « géométrie » et de «l'arithmétique » mais « rien qui se rapporte aux dispositions, aux actions, aux passions, et aux intérêts des hommes ${ }^{22} »$.

Pour beaucoup d'auteurs contre-révolutionnaires, le mot «système» devient synonyme de folie. Ainsi Mallet du Pan écrit-il en 1793: «Au sein des premières fureurs et des systèmes s'éleva ce club des Jacobins ${ }^{23}$. » Barruel s'interroge à propos des mêmes Jacobins en 1798 : «Qu'est-ce que cette secte dévorante ? D'où lui viennent à la fois et cet essaim d'adeptes, et ces systèmes et ce délire de rage contre tous les autels et tous les trônes ${ }^{24}$ ? » Après la chute de Robespierre, "système » devient l'un des mots clés visant à disqualifier radicalement la politique menée par le gouvernement révolutionnaire, notamment dans l'expression "système de la Terreur », utilisée pour la première fois dans ce sens par Barère à la Convention le 11 thermidor $^{25}$. Il est repris autant par les écrivains les plus hostiles à la Révolution que par ceux qui, comme Constant, ne la rejettent pas en bloc $^{26}$.

\footnotetext{
${ }^{20}$ Joseph de Maistre, Considérations sur la France, Londres, 1797, p. 37.

${ }^{21}$ Edmund Burke, Lettre de M. Burke à un membre de l'Assemblée nationale de France, Paris, Artaud, 1791, p. 44 et 77.

22 Edmund Burke, Réflexions sur la révolution de France, Paris, Laurent fils, 1790, p. 390-391.

${ }^{23}$ Jacques Mallet du Pan, Considérations sur la nature de la révolution de France et sur les Causes qui en prolongent la durée, Londres, 1793 , p. 12.

24 Augustin Barruel, Mémoires pour servir à l'bistoire du jacobinisme, Hambourg, P. Fauche, 1798, vol. 1, p. vi.

25 Voir Jean-Clément Martin, Violence et Révolution. Essai sur la naissance d'un mythe national, Paris, Seuil, 2006, p. 216.

26 Benjamin Constant, Des effets de la Terreur, [1797] dans De la force du gouvernement actuel de la France et de la nécessité de s'y rallier, Des réactions politiques, Des effets de la terreur, éd. de Philippe Raynaud, Paris, Flammarion, Champs, 1988, p. $166:$ : la terreur, réduite en système, et justifiée sous cette forme, est beaucoup plus horrible que la violence féroce et brutale des terroristes ».
} 
Liés à la fois par l'idée d'abstraction et par l'idée d'excès, système et Terreur sont de plus en plus souvent associés. Dans une même logique, bien des auteurs associent Révolution et philosophie, accusant cette dernière d'être la principale responsable des malheurs révolutionnaires. Une vingtaine de pages seulement après avoir rêvé de tableaux mathématiques, Chateaubriand écrit dans son Essai sur les Révolutions que «le vrai esprit des Encyclopédistes était une fureur persécutante de systèmes ${ }^{27} »$. En 1802, dans un texte de ralliement à Napoléon précisément intitulé Du 18 Brumaire, opposé au système de la Terreur, Joseph Fiévée précise l'accusation :

L'esprit d'analyse est destructif de sa nature, car on n'analyse qu'en décomposant ; toutes les fois que, dans un pays, cet esprit sera dominant, il s'attachera à tout, il décomposera tout, la grandeur, la gloire, la politique, les mœurs et les lois. L'esprit de la haute littérature au contraire, tous les arts d'imagination, sont essentiellement créateurs et vivifiants ${ }^{28}$.

La critique de l'esprit de système contribue ainsi à l'opposition durable qui s'invente alors : la philosophie, rejetée du côté des sciences, est séparée de la littérature, spécialisée dans ce qui ne se mesure pas et seule force de vie véritable. L'évolution de Chateaubriand est significative: dès la fin de l'Essai il rejette les sciences et trouve refuge dans la nature sauvage du nord de l'Amérique. Dans Le Génie du christianisme, il met définitivement les beautés poétiques au premier plan.

La volonté de fonder une pensée conservatrice par les sciences se heurte ainsi à la critique de l'esprit de système. Bonald, dont les textes affichent toujours une forte prétention scientifique, donne un exemple significatif de cette contradiction. En 1800 encore, dans son Essai analytique sur les lois naturelles de l'ordre social, le modèle scientifique qu'il entend appliquer au «monde moral» est explicitement celui de la physique newtonienne : «Ce sont [les] lois générales du monde moral que j’ai cherché à déterminer [...] comme Kepler et Newton ont cherché à découvrir et à calculer les lois générales du monde physique, lois générales de l'ordre entre les être moraux, auxquelles la société obéit $^{29}$. » Quatre ans plus tôt en revanche, lorsqu'il insère des «Observations » sur l'Esquisse de Condorcet à la fin du deuxième tome de sa Théorie du pouvoir politique et religieux, il établit des distinctions très marquées entre les sciences naturelles et les sciences humaines. Les premières ont pour objet ce qui peut être "pesé, mesuré, calculé », les autres, tout ce qui échappe à ces opérations: "règles des mœurs et des devoirs", "principes de l'ordre social », «vérité », «vertu » et « bonheur ${ }^{30}$ ». La découverte du texte de Condorcet conduit ainsi Bonald à faire une critique précise et sévère d'analogies dont il use lui-même volontiers. Trois raisons expliquent qu'il combatte ainsi l'idée de calcul, alors même que dans la préface du premier volume il dit vouloir retrouver du côté de la politique et de la morale «l'évidence du calcul ${ }^{31} »$. D'une part l'idée de calcul telle que l'utilise Condorcet unifie tous les savoirs au point de menacer la séparation entre l'âme et le corps, le créateur et la création. Le contexte institutionnel explique également la réaction de Bonald au texte de Condorcet: la République combattue par l'auteur de la Théorie du ponvoir politique et religieux prend alors la forme d'un pouvoir dont l'objectif principal est de soumettre la politique au calcul. Le développement de l'administration et la création de l'Institut, avec sa classe de sciences politiques et morales, sont deux aspects de cette évolution administrative qu'un défenseur du trône et de l'autel ne saurait encourager, fût-ce indirectement, en donnant trop souvent à penser que la nature et les sociétés humaines obéissent aux mêmes

${ }^{27}$ Chateaubriand, op. cit., p. 1096.

28 Joseph Fiévée, Du 18 Brumaire, opposé an système de la Terreur, Paris, Maradan, an X-1802, note 1, p. 13.

${ }^{29}$ Louis de Bonald, Essai analytique sur les lois naturelles de l'ordre social, 1800, vol. 2, p. 12.

${ }^{30}$ Louis de Bonald, Théorie du pouvoir politique et religieux, dans la société civile, démontrée par le raisonnement et par l'bistoire, 1796, vol. 2, p. 498.

31 Ibid., vol. 1, p. ix. 
lois. Enfin, le calcul de Condorcet n'est pas un instrument de mesure qui viendrait établir des règles immuables comme celles de Newton mais un instrument de prospective : il ne s'agit pas d'un calcul régulateur, qui limite l'action et rend évident la nécessité de se plier à ce qui est voulu par Dieu, mais d'un calcul qui invite à des actions nouvelles.

Cet aspect de la pensée de Condorcet est un exemple des évolutions à l'œuvre chez les défenseurs du progrès. S'interrogeant sur les conditions de possibilité de sciences humaines ou politiques, ils développent une pensée de moins en moins systématique et mettent à distance l'idée de certitude scientifique. Le modèle mathématique des calculs de probabilité permet ainsi à Condorcet de développer une science des incertitudes sociales :

L'application du calcul des combinaisons et des probabilités à ces mêmes sciences, promet des progrès d'autant plus importants, qu'elle est à la fois le seul moyen de donner à leurs résultats une précision presque mathématique, et d'en apprécier le degré de certitude ou de vraisemblance ${ }^{32}$.

Sans rien atténuer de la positivité militante de Condorcet ${ }^{33}$, il faut remarquer l'adverbe «presque » dans l'expression « une précision presque mathématique » ainsi que la formule «le degré de certitude ou de vraisemblance ». Condorcet dit bien que l'homme ne pourra jamais tout calculer. C'est bien d'ailleurs ce qui garantit sa perfectibilité infinie : puisqu'il n'aura jamais fini de calculer et d'apprendre, il calculera et apprendra toujours davantage.

Dans ses Leçons d'histoire, Volney définit l'histoire comme "un art systématique de calculs qui ne sont que probables ${ }^{34} »$. Il esquisse le programme d'une histoire digne d'être considérée comme une science politique lorsqu'il propose de pratiquer lui aussi les « hautes mathématiques de l'Histoire ${ }^{35}$ ", c'est-à-dire d'interroger méthodiquement le degré de certitude dont l'histoire est susceptible. Pour les besoins de sa démonstration, Volney oppose l'histoire aux « autres sciences » enseignées à l'École normale. Sa réflexion s’inscrit pourtant dans une évolution qui touche les sciences physiques. Les modèles proposés par les sciences du vivant prennent de plus en plus d'importance : une pensée que l'on pourra bientôt qualifier de biologique se développe au détriment de conceptions systématiques ou mécanistes. Cette évolution permet au naturaliste Lamarck de se livrer à la critique de l'esprit de système lorsqu'il veut disqualifier la pensée de ses prédécesseurs et souligner ce qui fait l'originalité de ses propres travaux dans un texte de 1806 :

Maintenant, on ne fait plus de cas en France de ces systèmes artificiels fondés sur des caractères qui compromettent les rapports naturels entre les objets qui y sont soumis ; systèmes qui donnaient lieu à des distributions et des divisions nuisibles à nos connaissances de la nature 36

Bien qu'elle ait fait l'objet d'innombrables analyses, la Révolution n'a que très rarement été réduite en système par ses contemporains. Le volontarisme scientifique des défenseurs du progrès ne saurait être ramené au désir de système qu'expriment quelques

\footnotetext{
32 Condorcet, op. cit., p. 361-362.

33 Le volontarisme scientifique et politique de Condorcet, si sensible dans l'Esquisse, a souvent été souligné. Jean Starobinski, citant ce passage, insiste sur les assurances nouvelles que le calcul donnera à l'humanité : "Condorcet annonce un âge où l'action politique pourra, grâce au calcul, anticiper et atteindre plus sûrement ses buts " (Jean Starobinski, Action et Réaction, vie et aventure d'un couple, Paris, Seuil, 1999, p. 297). Keith M. Baker met l'accent sur les « énormes possibilités » du «calcul des probabilités » (Keith Michael Baker, Condorcet. Raison et politique, Paris, Hermann, 1988, p. 499).

34 Volney, Leçons d'bistoire prononcées à l'École normale en l'an III de la république française, Paris, J. A. Brosson, an VIII, p. 140141.

35 Ibid., p. 104.

36 Jean-Baptiste Lamarck, "Discours d'ouverture du cours des animaux sans vertèbres, prononcé dans le Muséum d'histoire naturelle, en mai1806» dans Discours d'ouverture, éd. de Alfred Giard, Lille, impr. de L. Danel, 1907, p. 114.
} 
citations isolées. Les textes de Condorcet, de Constant, de Volney et de Mme de Staël héritent de toute la pensée antisystématique du siècle des Lumières. Inventant des outils conceptuels d'explication et de prospective, ils développent des sciences de l'incertitude et de l'évolution, à l'opposé des systèmes réguliers où tout se répète et peut être calculé avec certitude. Les sciences naturelles évoluent pour une part dans la même direction, avec le développement des sciences du vivant ou l'introduction par Laplace d'une dimension historique nouvelle dans l'astronomie. Ainsi, la Révolution n'entraîne pas un retour à l'esprit de système, par volonté de ramener le chaos à un ordre rationnel, mais bien plutôt un renouveau des pensées scientifiques non systématiques.

La tentation de l'esprit de système est plus forte pour les auteurs les plus réactionnaires: en promouvant une pensée systématique qu'ils présentent comme un héritage $\mathrm{du} \mathrm{XVII}^{\mathrm{e}}$ siècle, ils entendent revenir à une science fantasmée où tout serait cohérent, dans un univers clos où les révolutions ne remettent pas l'ordre général en cause. Temps de vérité et de certitude, le XVII ${ }^{\mathrm{e}}$ siècle de Newton fait ressortir par contraste les errances et les erreurs du XVIII ${ }^{e}$ siècle. Mais les tentatives de réduire la Révolution en système sont comme minées de l'intérieur par l'usage très politique qui est fait de la critique de l'esprit de système. Le mot système devient un marqueur politique, étroitement associé aux mots philosophie, révolution et terreur. C'est en cela sans doute que la Révolution a joué le plus grand rôle, avec des conséquences lourdes sur les représentations de la littérature qui s'imposent alors et dont nous sommes encore, pour une part, les héritiers. La pensée du $\mathrm{XVIII}^{\mathrm{e}}$ siècle a été réduite à l'expression d'une raison présentée à tort comme systématique. La littérature telle qu'elle s'est inventée dans les premières années du XIX ${ }^{e}$ siècle en serait l'antithèse.

Olivier Ritz

Université Paris-Sorbonne 